\title{
(1) nasen \\ Response to the NCEPOD report: development of a care bundle for patients admitted with decompensated cirrhosis-the first 24 h
}

\author{
Stuart McPherson, ${ }^{1,2}$ Jessica Dyson, ${ }^{1,2}$ Andrew Austin, ${ }^{3,4}$ \\ Mark Hudson ${ }^{1,2,5}$
}

For numbered affiliations see end of article.

\section{Correspondence to}

Dr Stuart McPherson, Liver Unit, Freeman Hospital, High Heaton, Newcastle upon Tyne, NE7 7DN, UK: stuart.mcpherson@nuth.nhs.uk

Received 23 June 2014 Revised 22 September 2014 Accepted 28 September 2014 Published Online First 2 December 2014

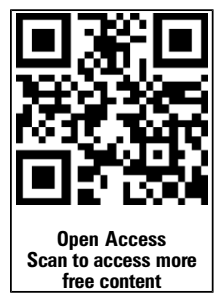

CrossMark

To cite: McPherson $S$, Dyson J, Austin A, et al. Frontline Gastroenterology 2016;7:16-23.

\section{ABSTRACT}

Recently, there has been a significant increase in the prevalence of chronic liver disease in the UK, and as a result, hospital admissions and deaths due to liver disease have also increased. The 2013 National Confidential Enquiry into Patient Outcome and Death (NCEPOD) of patients with alcohol-related liver disease (ARLD) found that less than half the number of patients who died from ARLD received 'good care', and avoidable deaths were identified. In order to improve the care of patients admitted with ARLD, the NCEPOD report recommended that a 'toolkit' for the acute management of patients admitted with decompensated ARLD be developed and made widely available. As a result, we have developed a 'care bundle' for patients admitted with decompensated cirrhosis (of all aetiologies) to ensure that effective evidence-based treatments are delivered within the first $24 \mathrm{~h}$. This care bundle provides a checklist to ensure that all appropriate investigations are undertaken when a patient with decompensated cirrhosis presents and provides clinicians with clear guidance on the initial management of alcohol withdrawal, infection, acute kidney injury, gastrointestinal bleeding and encephalopathy. The first $24 \mathrm{~h}$ are particularly important, as early intervention can reduce mortality and shorten hospital stay, and specialist gastroenterology/liver advice is not always available during this period. This review will discuss the care bundle and the evidence base behind the treatment recommendations made.

\section{INTRODUCTION}

Over the last 20 years, there has been a significant increase in the prevalence of chronic liver disease in the UK, with the major causes being alcohol-related liver disease (ARLD), hepatitis B and C and obesity-related liver disease. ${ }^{1}$ There has also been a substantial rise in hospital admissions with complications of liver disease, as well as a steady rise in liverrelated deaths in the UK. ${ }^{12}$ Liver disease is now one of the major causes of premature death in the UK. ${ }^{3}$ Decompensated cirrhosis and acute on chronic liver failure are common causes for hospital admission and are associated with a high mortality rate. ${ }^{4}$ The 2013 National Confidential Enquiry into Patient Outcome and Death (NCEPOD) of patients with ARLD 'Measuring the Units' raised concerns about suboptimal care of patients in hospital with complications of cirrhosis (due to ARLD), which might have contributed to the rise in liver-related mortality. ${ }^{5}$ The report found that less than half $(47 \%)$ the patients who died from ARLD received 'good care', and avoidable deaths were identified. It is likely that similar findings would be observed for the management of patients with cirrhosis of other aetiologies.

In order to improve the care of patients admitted with ARLD, the NCEPOD report recommended that a 'toolkit' for 


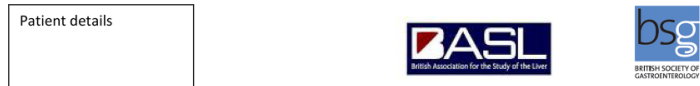

Decompensated Cirrhosis Care Bundle - First 24 Hours

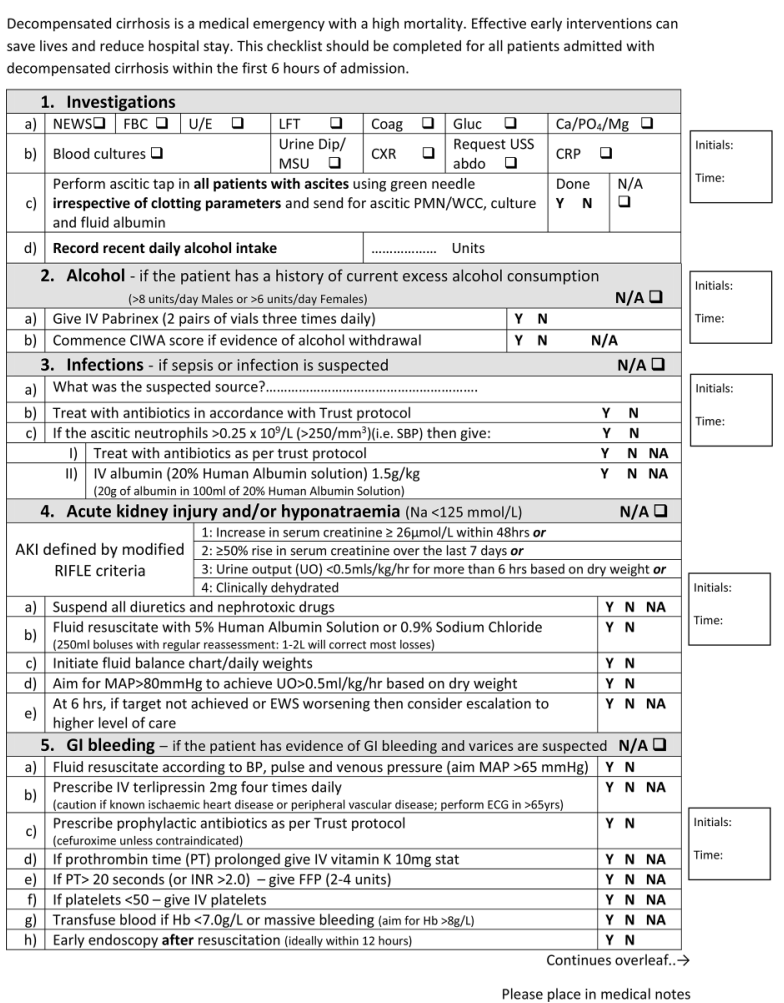

Please place in medical notes

Figure 1 The care bundle for decompensated cirrhosis.

the acute management of patients admitted with decompensated ARLD be developed and made widely available..$^{5}$ As a result, we have developed a 'care bundle' for patients admitted with decompensated cirrhosis (of all aetiologies) to ensure that effective evidence-based treatments are delivered within the first $24 \mathrm{~h}$. The first $24 \mathrm{~h}$ are particularly important as early intervention for presentations such as sepsis, bleeding or renal dysfunction in patients with cirrhosis can reduce mortality and shorten hospital stay, and specialist gastroenterology/liver advice is not always available during this period. This review will discuss the care bundle and the evidence base behind the treatment recommendations made. A copy for general use is available at http://www.nescn.nhs.uk/wp-content/ uploads/2014/05/Cirrhosis-Care-Bundle.pdf and the care bundle is shown in figure 1 .

\section{THE CARE BUNDLE}

The care bundle is designed to be completed for all patients presenting with decompensated cirrhosis and should be commenced within $6 \mathrm{~h}$ of admission. 'Decompensated cirrhosis' is defined as an acute deterioration in liver function in a patient with cirrhosis that can manifest with the following: jaundice, increasing ascites, hepatic encephalopathy, renal impairment/ hypovolaemia, gastrointestinal (GI) bleeding, or signs
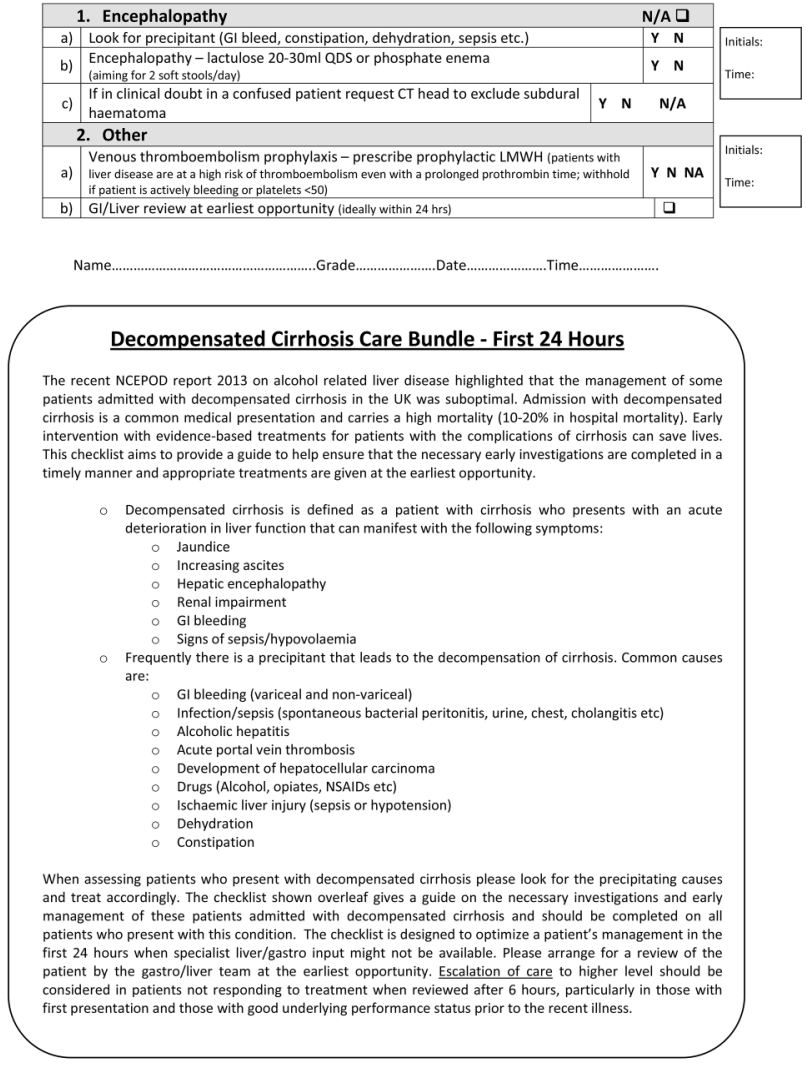

of sepsis. Common precipitants for decompensated cirrhosis are shown in box 1 . This care bundle is primarily designed to provide recommendations on management for the first $24 \mathrm{~h}$ only. After this period, it is expected that specialist GI/liver input will be available to provide guidance on further management.

\section{BASELINE INVESTIGATIONS}

On admission, it is vital that patients presenting with decompensated cirrhosis have a full history and clinical examination to look for the cause of deterioration in liver function, infection or evidence of GI bleeding. The National Early Warning Score should be recorded and used to monitor the patient's physiological status. ${ }^{6}$ Blood tests should be taken to assess liver and renal function, and this should include: full blood count, urea and electrolytes (including calcium/phosphate/magnesium), liver function tests, coagulation profile and glucose. As sepsis is a frequent cause of admission, patients should also be screened for infection with clinical examination, urinalysis and urine culture, chest X-ray, blood cultures and C-reactive protein (CRP). ${ }^{5} 7$ Although not always a routine test, serum CRP levels can help detect infection in cirrhotic patients early. ${ }^{8}$ One study showed that a CRP level greater than $10 \mathrm{mg} / \mathrm{L}$ was predictive of infections in patients with cirrhosis (area under the receiver 
Box 1 Definition of decompensated cirrhosis and common precipitants

Decompensated cirrhosis=deterioration in liver function that can manifest with the following:

- Jaundice

- Increasing ascites

- Hepatic encephalopathy

- Renal impairment/hypovolaemia

Signs of sepsis/hypovolaemia

Common precipitants of decompensated cirrhosis:

- Gastrointestinal bleeding (variceal and non-variceal)

- Infection/sepsis (spontaneous bacterial peritonitis, urine, chest, cholangitis, etc)

- Alcoholic hepatitis

- Acute portal vein thrombosis

- Development of hepatocellular carcinoma

- Drugs (alcohol, opiates, NSAIDs, etc)

- Ischaemic liver injury (sepsis or hypotension)

- Dehydration

- Constipation (encephalopathy)

operator curve 0.93 , sensitivity $84 \%$, specificity $91 \%$ ), although the CRP can be elevated for other reasons, such as alcoholic hepatitis. ${ }^{9}$

Additionally, an ascitic tap should be performed for all patients who have clinically detectable ascites to exclude spontaneous bacterial peritonitis (SBP). ${ }^{10}$ Coagulopathy is not a contraindication to this procedure, ${ }^{5}$ and measures to correct coagulopathy are NOT required. Ascitic fluid should be sent to the lab for analysis of the fluid: polymorphonuclear (PMN) and white cell count, microscopy and culture, protein and albumin content. ${ }^{11}$ An abdominal ultrasound should be requested and performed at the earliest opportunity (unless an ultrasound has been conducted in the last month), ideally within $24 \mathrm{~h}$, and should include an assessment of portal vein patency.

\section{Alcohol}

Seventy per cent of patients in the UK, admitted with cirrhosis, have alcohol as the major aetiological factor. ${ }^{12}$ The patient's current and previous alcohol history (in units/day: box 2), time of last drink and any symptoms of alcohol withdrawal should be documented. ${ }^{5}$ For

\section{Box 2 Definition of units of alcohol}

- One unit of alcohol $=10 \mathrm{~mL}$ of alcohol or $8 \mathrm{~g}$ of alcohol

- Example: a $500 \mathrm{~mL}$ can of $5 \%$ Beer $=25 \mathrm{~mL}$ of alcohol=2.5 units of alcohol

- The maximum recommended weekly alcohol consumption is 21 units for men and 14 units for women. patients with current excessive alcohol consumption, parenteral thiamine and other B vitamins (Pabrinex: two pairs of vials three times daily for 3 days) should be given to treat thiamine deficiency and reduce the risk of Wernicke's encephalopathy or Korsakoff's syndrome. ${ }^{13}$ Patients who have symptoms of alcohol withdrawal or are at high risk of alcohol withdrawal, seizures or delirium tremens should be assessed with a validated tool for alcohol withdrawal, such as the revised Clinical Institute Withdrawal Assessment-Alcohol (CIWA-Ar) scale, and be treated with a symptom-triggered regimen of benzodiazepines. ${ }^{13} 14$

\section{Infections}

Patients with cirrhosis have impaired defence against bacteria due to immune dysfunction, and as a result, bacterial infections are one of the most frequent reasons for admission and are associated with a high mortality rate. ${ }^{815} 16$ A careful assessment for infection is critical in all patients admitted with decompensated cirrhosis, as prompt treatment with antibiotics improves prognosis. ${ }^{17}$ The most common infections in cirrhotics are SBP, followed by urinary tract infections and pneumonia, ${ }^{8} 18$ and Gram-negative bacilli, such as Escherichia coli, are the most frequently encountered pathogens. ${ }^{19}$ There has been a recent increase in the prevalence of infections with multidrug resistant bacteria in patients with cirrhosis, which emphasises the importance of taking the appropriate samples for culture and sensitivity, so patients can have their antibiotics modified accordingly. ${ }^{20}$ Importantly, patients with cirrhosis do not always display typical signs of infection, such as pyrexia or rise in CRP, so clinicians must have a high index of suspicion for infection. Once suspected clinically, infection should be treated promptly with broad spectrum antibiotics as per hospital protocol.

SBP is defined as infection of the ascitic fluid in the absence of a secondary cause, such as intestinal perforation, and occurs in approximately $10 \%$ of hospitalised patients with cirrhosis. ${ }^{16}{ }^{18}$ Although abdominal pain and fever are commonly seen in patients with SBP, symptoms are frequently absent. ${ }^{21}$ It is, therefore, recommended that all patients presenting with ascites have a diagnostic ascitic tap to exclude SBP on admission to hospital, or if there is deterioration in their clinical status. ${ }^{5} 1011$ Diagnosis of SBP is made when the absolute number of PMN cells is $>250 / \mathrm{mm}^{3}$ of ascitic fluid. ${ }^{22}$ Upon diagnosis, SBP should be empirically treated with broad spectrum antibiotics, such as third-generation cephalosporin (cefotaxime or ceftriaxone), co-amoxiclav or ciprofloxacin, according to hospital policy, with modifications made in light of subsequent culture results. ${ }^{8} 1011$ Patients with SBP are at high risk of developing hepatorenal syndrome (HRS) and should have intravenous albumin administered to prevent worsening of renal function. ${ }^{10}$ Studies have shown that intravenous albumin $1.5 \mathrm{~g} / \mathrm{kg}$ 
at diagnosis and $1 \mathrm{~g} / \mathrm{kg}$ at $72 \mathrm{~h}$ reduces the incidence of HRS from $30 \%$ to $10 \%$ and reduces mortality from $29 \%$ to $10 \% .^{23}$

\section{Acute kidney injury and/or hyponatraemia}

Acute kidney injury (AKI) is estimated to occur in approximately $20 \%$ of hospitalised patients with cirrhosis, and is associated with a poor prognosis. ${ }^{24} 25$ Several definitions of AKI exist, but the National Institute of Health and Care Excellence (NICE) recommends the use of the modified RIFLE (Risk Injury Failure Loss and End stage renal disease) criteria that were devised by the Acute Kidney Injury Network to detect subjects at risk of AKI. ${ }^{26}{ }^{27}$ AKI is defined if any of the following criteria are met: (1) an absolute rise in serum creatinine of $\geq 26 \mu \mathrm{mol} / \mathrm{L}$ within $48 \mathrm{~h}$; (2) a $\geq 50 \%$ increase in serum creatinine known or presumed to have occurred in the last 7 days; (3) a fall in urine output to $<0.5 \mathrm{~mL} / \mathrm{kg} / \mathrm{h}$ for 6 h. ${ }^{27}$ It should be noted that patients with cirrhosis frequently have low baseline creatinine levels, as many have reduced muscle bulk and malnutrition. Therefore, some patients with creatinine levels within the normal range can have a significantly reduced glomerular filtration rate.

AKI in patients with cirrhosis is commonly multifactorial, but prerenal AKI is most common (45\%), followed by acute tubular necrosis and glomerulonephritis (32\%), HRS $(23 \%)$ and rarely postrenal $(<1 \%){ }^{25}$ It is important to identify the cause of AKI, as the underlying cause has implications on prognosis. Type 1 HRS (acute) carries a particularly poor prognosis with mortality rates approaching 100\% without treatment. HRS is a diagnosis of exclusion, and current diagnostic criteria are shown in box $3 .^{28}$ It is important to note that before a diagnosis of HRS can be made patients need to have their diuretics and nephrotoxic medications suspended and be volume expanded with albumin, so a diagnosis of HRS can usually not be made within the first $24 \mathrm{~h}$. If HRS is subsequently

\section{Box 3 Diagnostic criteria for hepatorenal syndrome ${ }^{28}$}

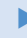

Cirrhosis with ascites

Serum creatinine $>133 \mu \mathrm{mol} / \mathrm{L}$

- No improvement of serum creatinine to $<133 \mu \mathrm{mol} / \mathrm{L}$ after at least 2 days with diuretic withdrawal and volume expansion with albumin (1 $\mathrm{g} / \mathrm{kg}$ up to a maximum of $100 \mathrm{~g} /$ day)

- Absence of shock

- No current treatment with nephrotoxic drugs

- Absence of parenchymal kidney disease as indicated by proteinuria ( $>500 \mathrm{mg} /$ day) or microhaematuria (>50 red blood cells per high-power field) and/or a normal renal ultrasonography diagnosed, patients should be treated with intravenous albumin and terlipressin, as this has been shown to reduce mortality in patients with HRS. ${ }^{29}$

Early intervention in patients with AKI can prevent further deterioration in renal function leading to renal failure. Initial management of AKI should include suspension of all diuretics and nephrotoxic drugs. ${ }^{30} 31$ Patients should be fluid-resuscitated with $0.9 \%$ saline or $5 \%$ albumin, giving boluses of $250 \mathrm{~mL}$ with regular volume status reassessment aiming to achieve euvolaemia with a urine output of $>0.5 \mathrm{~mL} / \mathrm{kg} / \mathrm{h}$ based on dry weight. ${ }^{32} 33$ Accurate fluid balance monitoring and daily weight charts should be commenced. Usually 1-2 L of intravenous fluid will correct most losses and render the patient euvolaemic. If after $6 \mathrm{~h}$ the target urine output is not achieved, or the patient's clinical parameters are worsening, then escalation to high-dependency care for more invasive monitoring, inotropic support or renal support should be considered.

Hyponatraemia is common in patients with decompensated cirrhosis and can be caused by multiple factors including: hormonal dysregulation of salt and water balance in response to portal hypertension, dehydration, treatment with diuretics, infections and excessive use of hypotonic fluids, such as $5 \%$ dextrose. ${ }^{10}$ Patients with serum sodium levels $<125 \mathrm{mmol} / \mathrm{L}$ are at risk of seizures. A careful history, clinical examination, medication review and review of other biochemistry are essential to determine the cause of hyponatraemia. Assessment of the patient's volume status is fundamental, and subjects who are hypovolaemic should be fluid-resuscitated. Patients with hypervolaemic hyponatraemia should be treated with fluid restriction. However, it should be noted that some patients with significant ascites, peripheral oedema and low serum albumin levels who appear hypervolaemic are actually intravascularly deplete, and treatment with intravenous albumin can correct the hyponatraemia. ${ }^{34}$ Correction of hyponatraemia should be slow $(<10 \mathrm{mmol} / \mathrm{L}$ increase per $24 \mathrm{~h})$, as rapid changes in serum sodium can precipitate osmotic demyelination syndrome.

\section{GI bleeding}

Bleeding oesophageal and gastric varices are another important complication of cirrhosis with a high mortality rate. A recent UK-wide audit showed the overall 30 -days mortality was $15 \%$ for patients with acute variceal bleeding, with higher mortality rates in subjects with more advanced cirrhosis. ${ }^{35}$ Airway protection is vital, particularly in patients with encephalopathy or massive GI bleeding to reduce the risk of aspiration. ${ }^{36}$ In patients with known varices or portal hypertension, upper GI bleeding should be regarded as variceal until proven otherwise, although a significant proportion will have non-variceal bleeding. Patients should be fluid-resuscitated according to pulse and blood pressure, aiming for a mean arterial pressure of $>65 \mathrm{~mm} \mathrm{Hg}^{7}$ 
Transfusion of red blood cells is indicated when the haemoglobin $(\mathrm{Hb})$ falls below $7 \mathrm{~g} / \mathrm{dL}^{37}$ aiming for an $\mathrm{Hb}$ of $8 \mathrm{~g} / \mathrm{dL}$. It has been recently shown that a restrictive blood transfusion strategy (transfuse when $\mathrm{Hb}<7 \mathrm{~g}$ / dL) had lower mortality compared with a liberal strategy of transfusion (transfuse when $\mathrm{Hb}<9 \mathrm{~g} / \mathrm{dL}$ ) in patients with cirrhosis and with GI bleeding. ${ }^{38}$ The liberal strategy also increased the portal pressure over 5 days, thus increasing the potential for rebleeding. ${ }^{38}$ Coagulation abnormalities are common in patients with cirrhosis and may require correction in patients with active bleeding. Although not evaluated in randomised controlled trials, correction of blood clotting abnormalities is recommended by NICE when the international normalised ratio (INR) is $>1.5$ (fresh frozen plasma), platelet count is $<50 \times 10^{9} / \mathrm{L}$ (platelets) or fibrinogen $<1.0 \mathrm{~g} / \mathrm{L}$ (cryoprecipitate). ${ }^{39}$ Consider giving intravenous vitamin $\mathrm{K}$ in patients with cirrhosis and with a prolonged prothrombin time, as vitamin $\mathrm{K}$ deficiency is common due to malnutrition and chronic cholestasis.

In patients with suspected variceal bleeding, terlipressin ( $2 \mathrm{mg}$ four times daily) should be administered intravenously as soon as possible to control bleeding. ${ }^{39}$ Terlipressin is a vasopressin analogue that reduces portal pressure by inducing splanchnic vasoconstriction and has been shown to improve control of bleeding, as well as reduce all-cause mortality in patients with cirrhosis and with variceal bleeding. ${ }^{40} 41$ Significant adverse events with terlipressin are uncommon, but because of its vasoconstrictor activity it should be used with caution in patients with known or suspected ischaemic heart disease or peripheral vascular disease and those older than 65 years of age, making a baseline ECG mandatory. For patients who have a contraindication to terlipressin (or if terlipres$\sin$ is unavailable), a continuous intravenous infusion of octreotide is an alternative. ${ }^{37} 40$ Once patients have been adequately resuscitated, endoscopy should be performed at the earliest opportunity (ideally within 6-12 h, but not more than $24 \mathrm{~h}$ ) to determine the cause of bleeding and perform endotherapy as indicated. ${ }^{7} 39$ Bacterial infections, particularly SBP, are common in patients with cirrhosis in the first 7 days post-GI bleeding, occurring in 25\%-65\% of patients. ${ }^{10}$ Prophylaxis with antibiotics post-GI bleed have been shown to reduce short-term mortality, as well as the risk of rebleeding, septicaemia and $\mathrm{SBP}^{42}{ }^{43}$ It is, therefore, recommended that patients with cirrhosis and GI bleeding (variceal and nonvariceal) receive prophylaxis with a broad spectrum antibiotic, such as cefotaxime or co-amoxiclav, as per hospital protocol, as soon as possible. ${ }^{8} 39$ Patients with advanced cirrhosis (any two of the following: ascites, severe malnutrition, encephalopathy or jaundice) are at highest risk of developing infection and should receive the antibiotics intravenously. ${ }^{44}$ Importantly, there appears to be no increase in the incidence of Clostridium difficile infection in patients receiving antibiotic prophylaxis for variceal bleeding compared with the hospital medical population. ${ }^{45}$

\section{Encephalopathy (delirium)}

Encephalopathy is another common complication in patients with cirrhosis. ${ }^{46}$ A careful assessment of the cause of encephalopathy should be undertaken looking for causes such as infection, electrolyte disturbance, occult bleeding, constipation or sedative drugs. ${ }^{7}$ For patients with grade 4 encephalopathy (coma) consider orotracheal intubation to reduce the risk of developing aspiration pneumonia. In conscious patients, oral lactulose $(20 \mathrm{~mL}-30 \mathrm{~mL}$ four times per day) should be administered aiming for 2 soft stools per day. For patients with a reduced conscious level, phosphate enemas or lactulose administered by nasogastric tube should be considered. Consider a CT head examination in patients where the confusion is unexplained to exclude intracranial pathology, such as subdural haematoma.

\section{Other considerations}

In the recent UK audit of blood component use in cirrhosis, thrombotic events occurred in $3 \%$ of patients. ${ }^{12}$ Thromboprophylaxis with low-dose, low molecular weight heparin (LMWH), is therefore recommended to reduce the risk of thrombosis, unless the patient is bleeding or has a platelet count less than $50 \times 10^{9} / \mathrm{L}^{12}$ Recent evidence suggests that even with apparent abnormalities on blood testing suggestive of a hypocoagulable state (prolonged prothrombin time), patients with cirrhosis typically have normal clot-forming capacity provided the platelet count is $>50 \times 10^{9} / \mathrm{L}$, and some patients with advanced cirrhosis are actually hypercoagulable. ${ }^{47}$ Changes in the balance of the hepatic production of procoagulant and anticoagulant blood clotting factors in cirrhosis are believed to be responsible for this phenomenon. ${ }^{48}$ Although thromboprophylaxis with LMWH has not been specifically trialled in hospitalised patients with decompensated cirrhosis to prevent thromboembolism, a previous study demonstrated that the administration of enoxaparin $4000 \mu /$ day for 48 weeks reduced the risk of portal vein thrombosis, liver decompensation and mortality, and no haemorrhagic side effects were seen. ${ }^{49}$

Malnutrition and nutritional deficiency are common in hospitalised patients with cirrhosis. ${ }^{50}$ Moreover, reduced calorific intake in hospitalised patients with cirrhosis is an independent risk factor for short-term mortality. ${ }^{50}$ Therefore, a nutritional assessment should be performed early in the admission and, if indicated, nutritional supplementation (oral or nasogastric) should be prescribed. Close monitoring of electrolytes (phosphate, magnesium, potassium and calcium) is mandatory as refeeding syndrome is common. Low electrolyte levels should be corrected promptly. 
Patients with decompensated cirrhosis should be reviewed by a consultant at the earliest opportunity, ideally within $6 \mathrm{~h}$, but not more than $12 \mathrm{~h}$, as they typically have multiple medical needs and have a high mortality. ${ }^{5}$ Additionally, these patients should be reviewed by a specialist gastroenterologist/hepatologist, ideally within $24 \mathrm{~h}$, but not more than $72 \mathrm{~h}$ after admission to hospital. ${ }^{5}$ In order to facilitate this, it is recommended that every acute hospital should have a liver lead or 'champion' to try and improve care for patients with liver disease and improve pathways of care. ${ }^{3}$

Escalation to a higher level of care needs to be considered in all patients not responding to treatment when reviewed after $6 \mathrm{~h}$, particularly in patients with WHO performance status $0-1$ prior to the recent illness and those with first presentation. ${ }^{5}$ A Consultant-toConsultant discussion is recommended. Prognostic scores that are commonly used in intensive care units (ICU), such as the Sepsis-Related Organ Failure Assessment score, assessed at baseline and at $48 \mathrm{~h}$ can be effective in predicting mortality in patients with cirrhosis who are admitted to ICU. These scores could be used to help identify patients who would benefit from ICU care, as well as identify those where prolonged ICU treatment would be futile. ${ }^{5152}$

\section{CONCLUSIONS}

The recent NCEPOD report on ARLD highlighted that the management of some patients with decompensated cirrhosis in the UK was suboptimal. In order to address these concerns, we have developed a 'care bundle' for patients admitted with decompensated cirrhosis, to ensure the appropriate initial investigations and interventions are conducted at an early stage

\section{Significance of this study}

What is already known about the subject?

The NCEPOD report on deaths from alcohol related liver disease raised concerns about the suboptimal care of patients with complications of cirrhosis.

\section{What are the new findings?}

We have developed a care bundle for patients admitted with decompensated cirrhosis to ensure they receive appropriate early intervention for alcohol withdrawal, infections, acute kidney injury, gastrointestinal bleeding and encephalopathy.

How might this impact on clinical practice in the foreseeable future?

It is hoped that this care bundle will be implemented widely in hospitals that admit patients with liver disease and that it will improve the care of patients with decompensated cirrhosis. when specialist advice may not be available. It is hoped that this care bundle will help improve the care of patients admitted to hospital with decompensated cirrhosis across the UK, but this will need to be formally evaluated. This care bundle could also be a focus for audit, nationally regarding the management of patients with decompensated cirrhosis.

\section{Author affiliations}

${ }^{1}$ Liver Unit, Freeman Hospital, Newcastle upon Tyne Hospitals NHS Foundation Trust, Newcastle upon Tyne, UK

${ }^{2}$ Institute of Cellular Medicine, Newcastle University, Newcastle upon Tyne, UK

${ }^{3}$ Department of Gastroenterology, Derby Hospitals

NHS Foundation Trust, Derby, UK

${ }^{4}$ British Society of Gastroenterology, Liver Section, London, UK

${ }^{5}$ British Association for the Study of the Liver, London, UK

Acknowledgements We thank the members of the BSG Liver Section Committee and the North East and North Cumbria Hepatology Network for their comments and suggestions for the care bundle. This care bundle has been endorsed by the British Society of Gastroenterology Liver section and the British Association for the Study of the Liver (BASL).

Contributors SM and AA conceived the idea for the care bundle. SM developed the initial draft of the manuscript and the care bundle. The care bundle and manuscript were critically evaluated and revised by AA, JD and MH. SM is guarantor. All authors contributed to the manuscript and have approved the final version.

Competing interests None.

Provenance and peer review Not commissioned; externally peer reviewed.

Open Access This is an Open Access article distributed in accordance with the Creative Commons Attribution Non Commercial (CC BY-NC 4.0) license, which permits others to distribute, remix, adapt, build upon this work noncommercially, and license their derivative works on different terms, provided the original work is properly cited and the use is non-commercial. See: http://creativecommons.org/licenses/by$\mathrm{nc} / 4.0$ /

\section{REFERENCES}

1 NHS liver care. NHS Atlas of Variation for People with liver disease: reducing the unwanted variation to increase value and improve quality. Right Care 2013:1-141.

2 Thomson SJ, Westlake S, Rahman TM, et al. Chronic liver disease--an increasing problem: a study of hospital admission and mortality rates in England, 1979-2005, with particular reference to alcoholic liver disease. Alcohol Alcohol 2008;43:416-22.

3 A Time to act: Improving Health and Outcomes in Liver Disease. The national plan for liver services (UK) British Society of Gastroenterology; 2009:1-52.

4 Moreau R, Arroyo V. Acute on chronic liver failure: a new clinical entity. Clin Gastroenterol Hepatol Published Online First: 27 Feb 2014.

5 NCEPOD. "Measuring the Units"-a review of patients who died with alcoholic related liver disease. National Confidential Enquiry into Patient Outcome and Death (UK); 2013:1-25. 
6 Physicians RCo. National Early Warning Score (NEWS): standardising the assessment of acute illness in the NHS. 2012:1-47.

7 Gines P, Fernandez J, Durand F, et al. Management of criticallyill cirrhotic patients. J Hepatol 2012;56(Suppl 1):S13-24.

8 Jalan R, Fernandez J, Wiest R, et al. Bacterial infections in cirrhosis: a position statement based on the EASL Special Conference 2013. J Hepatol 2014;60:1310-24.

9 Papp M, Vitalis Z, Altorjay I, et al. Acute phase proteins in the diagnosis and prediction of cirrhosis associated bacterial infections. Liver Int 2012;32:603-11.

10 EASL. Clinical practice guidelines on the management of ascites, spontaneous bacterial peritonitis, and hepatorenal syndrome in cirrhosis. J Hepatol 2010;53:397-417.

11 Moore KP, Aithal GP. Guidelines on the management of ascites in cirrhosis. Gut 2006;55(Suppl 6):vi1-12.

12 Desborough M, Jairath V, Sekhar M, et al. Multi Regional Audit of Blood Component Use in Patients with Cirrhosis. NHS Blood and Transplant, 2014.

13 NICE Clinical Guideline 100: Alcohol use disorders. National Institute of Health and Clinical Excellence (UK); 2010:1-15.

14 Sullivan JT, Sykora K, Schneiderman J, et al. Assessment of alcohol withdrawal: the revised clinical institute withdrawal assessment for alcohol scale (CIWA-Ar). Br J Addict 1989;84:1353-7.

15 Rajkovic IA, Williams R. Abnormalities of neutrophil phagocytosis, intracellular killing and metabolic activity in alcoholic cirrhosis and hepatitis. Hepatology 1986;6:252-62.

16 Borzio M, Salerno F, Piantoni L, et al. Bacterial infection in patients with advanced cirrhosis: a multicentre prospective study. Dig Liver Dis 2001;33:41-8.

17 Pleguezuelo M, Benitez JM, Jurado J, et al. Diagnosis and management of bacterial infections in decompensated cirrhosis. World J Hepatol 2013;5:16-25.

18 Fernandez J, Navasa M, Gomez J, et al. Bacterial infections in cirrhosis: epidemiological changes with invasive procedures and norfloxacin prophylaxis. Hepatology 2002;35:140-8.

19 Wong F, Bernardi M, Balk R, et al. Sepsis in cirrhosis: report on the 7th meeting of the International Ascites Club. Gut $2005 ; 54: 718-25$.

20 Fernandez J, Acevedo J, Castro M, et al. Prevalence and risk factors of infections by multiresistant bacteria in cirrhosis: a prospective study. Hepatology 2012;55:1551-61.

21 Chinnock B, Afarian $\mathrm{H}$, Minnigan $\mathrm{H}$, et al. Physician clinical impression does not rule out spontaneous bacterial peritonitis in patients undergoing emergency department paracentesis. Ann Emerg Med 2008;52:268-73.

22 Rimola A, Garcia-Tsao G, Navasa M, et al. Diagnosis, treatment and prophylaxis of spontaneous bacterial peritonitis: a consensus document. International Ascites Club. J Hepatol 2000;32:142-53.

23 Sort P, Navasa M, Arroyo V, et al. Effect of intravenous albumin on renal impairment and mortality in patients with cirrhosis and spontaneous bacterial peritonitis. N Engl J Med 1999;341:403-9.

24 Scott RA, Austin AS, Kolhe NV, et al. Acute kidney injury is independently associated with death in patients with cirrhosis. Frontline Gastroenterol 2013;4:191-7.

25 Garcia-Tsao G, Parikh CR, Viola A. Acute kidney injury in cirrhosis. Hepatology 2008;48:2064-77.

26 NICE Clinical Guideline 169: Acute Kidney Injury. National Institute of Health and Clinical Excellence (UK); 2013:1-39.
27 Mehta RL, Kellum JA, Shah SV, et al. Acute Kidney Injury Network: report of an initiative to improve outcomes in acute kidney injury. Crit Care 2007;11:R31.

28 Angeli P, Sanyal A, Moller S, et al. Current limits and future challenges in the management of renal dysfunction in patients with cirrhosis: report from the International Club of Ascites. Liver Int 2013;33:16-23.

29 Gluud LL, Christensen K, Christensen E, et al. Systematic review of randomized trials on vasoconstrictor drugs for hepatorenal syndrome. Hepatology 2010;51:576-84.

30 KDIGO Clinical Practice Guideline for Acute Kidney Injury. Kidney Int 2012;2(Suppl):1-138.

31 Berry PA, Thomson SJ. The patient presenting with decompensated cirrhosis. Acute Med 2013;12: 232-8.

32 NICE Clinical Guideline 174: Intravenous fluid therapy in adults in hospital. National Institute for Health and Clinical Excellence (UK); 2013:1-36.

33 Lewington A, Kanagasundaram S. Clinical Practice Guidelines: Acute Kidney Injury. The Renal Association 2011:1-55.

34 McCormick PA, Mistry P, Kaye G, et al. Intravenous albumin infusion is an effective therapy for hyponatraemia in cirrhotic patients with ascites. Gut 1990;31:204-7.

35 Jairath V, Rehal S, Logan R, et al. Acute variceal haemorrhage in the United Kingdom: Patient characteristics, management and outcomes in a nationwide audit. Dig Liver Dis 2014;46:419-26.

36 Jalan R, Hayes PC. UK guidelines on the management of variceal haemorrhage in cirrhotic patients. British Society of Gastroenterology. Gut 2000;46(Suppl 3-4):III1-15.

37 de Franchis R, Baveno VF. Revising consensus in portal hypertension: report of the Baveno $\mathrm{V}$ consensus workshop on methodology of diagnosis and therapy in portal hypertension. J Hepatol 2010;53:762-8.

38 Villanueva C, Colomo A, Bosch A, et al. Transfusion strategies for acute upper gastrointestinal bleeding. N Engl J Med 2013;368:11-21.

39 NICE Clinical guideline 141: Acute upper gastrointestinal bleeding. National Institute of Health and Clinical Excellence (UK); 2012:1-24.

40 Wells M, Chande N, Adams P, et al. Meta-analysis: vasoactive medications for the management of acute variceal bleeds. Aliment Pharmacol Ther 2012;35:1267-78.

41 Levacher S, Letoumelin P, Pateron D, et al. Early administration of terlipressin plus glyceryl trinitrate to control active upper gastrointestinal bleeding in cirrhotic patients. Lancet 1995;346:865-8.

42 Chavez-Tapia NC, Barrientos-Gutierrez T, Tellez-Avila FI, et al. Antibiotic prophylaxis for cirrhotic patients with upper gastrointestinal bleeding. Cochrane Database Syst Rev 2010; (9):CD002907.

43 Bernard B, Grange JD, Khac EN, et al. Antibiotic prophylaxis for the prevention of bacterial infections in cirrhotic patients with gastrointestinal bleeding: a meta-analysis. Hepatology 1999;29:1655-61.

44 Fernandez J, Ruiz del Arbol L, Gomez C, et al. Norfloxacin vs ceftriaxone in the prophylaxis of infections in patients with advanced cirrhosis and hemorrhage. Gastroenterology 2006;131:1049-56; quiz 285.

45 Brown MR, Jones G, Nash KL, et al. Antibiotic prophylaxis in variceal hemorrhage: timing, effectiveness and Clostridium difficile rates. World J Gastroenterol 2010;16:5317-23. 
46 Leise MD, Poterucha JJ, Kamath PS, et al. Management of hepatic encephalopathy in the hospital. Mayo Clin Proc 2014;89:241-53.

47 Tripodi A, Mannucci PM. The coagulopathy of chronic liver disease. N Engl J Med 2011;365:147-56.

48 Gatt A, Riddell A, Calvaruso V, et al. Enhanced thrombin generation in patients with cirrhosis-induced coagulopathy. J Thromb Haemost 2010;8:1994-2000.

49 Villa E, Camma C, Marietta M, et al. Enoxaparin prevents portal vein thrombosis and liver decompensation in patients with advanced cirrhosis. Gastroenterology 2012;143:1253-60 e1-4.
50 Campillo B, Richardet JP, Scherman E, et al. Evaluation of nutritional practice in hospitalized cirrhotic patients: results of a prospective study. Nutrition 2003;19:515-21.

51 Vincent JL, Moreno R, Takala J, et al. The SOFA (Sepsis-related Organ Failure Assessment) score to describe organ dysfunction/failure. On behalf of the Working Group on Sepsis-Related Problems of the European Society of Intensive Care Medicine. Intensive Care Med 1996;22:707-10.

52 Cholongitas E, Betrosian A, Senzolo M, et al. Prognostic models in cirrhotics admitted to intensive care units better predict outcome when assessed at $48 \mathrm{~h}$ after admission. J Gastroenterol Hepatol 2008;23(8 Pt 1):1223-7. 